\title{
Modeling of Heat Transfer, Fluid Flow and Solidification Microstructure of Nickel-base Superalloy Fabricated by Laser Powder Bed Fusion
}

\author{
Y.S. Lee and W. Zhang* \\ Welding Engineering Program, Department of Materials Science and Engineering \\ The Ohio State University, Columbus, Ohio, 43221, USA \\ *Corresponding author, zhang.3978@osu.edu
}

\begin{abstract}
Laser-Powder Bed Fusion (L-PBF), an additive manufacturing process, produces a distinctive microstructure that closely resembles the weld metal microstructure but at a much finer scale. The solidification parameters, particularly temperature gradient and solidification rate, are important to study the as-built microstructure. In the present study, a computational framework with meso-scale resolution is developed for L-PBF of Inconel ${ }^{\circledR} 718$ (IN718), a Ni-base superalloy. The framework combines a powder packing model based on Discrete Element Method and a 3-D transient heat and fluid flow simulation.The latter, i.e.,the molten pool model, capturesthe interaction between laser beam and individual powder particles including free surface evolution, surface tension and evaporation. The solidification parameters,calculated from the temperature fields, are used to assess the solidification morphology and grain size using existing theoretical models. The IN718 coupon built by L-PBF are characterized using optical and scanning electron microscopies. The experimental data of molten pool size and solidification microstructureare compared to the corresponding simulation results.
\end{abstract}

\section{Introduction}

Laser-Powder Bed Fusion (L-PBF) is one of the direct metal additive manufacturing (AM) processes. During processing, a fast-scanning laser beam melts and fusespowderparticles on a layer by layer basis to build metallic parts directly from 3-D Computer-Aided Design (CAD) data [1]. Rapid heating and cooling are common in L-PBF due to the fast scanning speed and low power of the laser beam. As a result, the solidification microstructure is typically far from that formed under equilibrium conditions. Quantitative understandingof the effect of processing parameters on the solidification microstructure is essential toimprove themechanical properties of parts fabricated by L-PBF.

Complex heat transfer and molten metal flow in the molten pool have a significant effect on the temperature gradient and solidification rate, which in turn, strongly influence the solidification morphology, grain size and growth direction[2, 3]. Experimental measurement of those complex physical phenomena of heat transfer and fluid convection is challenging because the process is both highly transient (about $1 \mathrm{~m} / \mathrm{s}$ scanning speed) and localized (about $2 \times 10^{-4} \mathrm{~m}$ or $200 \mu \mathrm{m}$ melt pool width)[1, 4]. Physics based numerical models, validated using the experimental data, are increasingly used to probe the molten pool dynamics during AM processing. For accurate modeling of solidification microstructure, a numerical model needs todescribe the interaction between the laser beam and the powder particles. Given the small ratio of laser beam diameter (about $100 \mu \mathrm{m}$ ) to particle diameter (about 20 to $40 \mu \mathrm{m}$ ), there is only a limited number of powder particles that are directly subject to the 
laser irradiation at a given time. Powder packing information is thus an important parameter for powder bed AM. Indeed, it was shown experimentally the impact of packing features (e.g., particle size distribution and packing density) on final part properties $[5,6]$. Hence, simulation of melting and fusing of individual particles into the molten pool,resolvedboth spatially and temporally, is essential. Moreover, the molten pool surface location as a function of time is unknown a priori and needs calculated.

The packing information, a prerequisite for modeling molten pool dynamics, is calculated using algorithms varying from simplyassigning a pre-defined packing sequence (e.g. face-centered cubic)[7, 8], to geometry-based rain model for random packing [9], and finally tocomplex dynamic simulation considering particle to particle contact interactions using discrete element method (DEM)[10, 11]. For simplicity, the powder particles are generally approximated as solid spheres. The coordinates and diameters of spherical particlesare calculated and then imported as initial geometry into the molten pool physics models.

The molten pool physics modelsare based on the numerical solution of transport phenomena equations including mass, Navier-Stokes (momentum), and energy conservation equations $[7,11$, 12].Volume of Fluid method (VOF), a moving interface-capturing technique, is typically used to calculate the molten pool surface location. Very small mesh size (on the order of $1 \mu \mathrm{m}$ ) and fine time increment (on the order of $0.1 \mu \mathrm{s}$ ) are used, making them suitable to capture the high gradients of temperature and solidification rate around the molten pool. Two groups of numerical methods are commonly used to solve the conservation equations: the traditional finite difference/volume methods (FDM/FVM) and a newer Lattice Boltzmann Method (LBM). For instance,Körneret al. developed a2-D transient LBM modelto study melting and solidificationin randomly stacked powder layers[9].They showed that the melt pool characteristics were significantly influenced by laser power, scanning speed and powder packing density.3-D transientmodels based on $\operatorname{LBM}[13], \operatorname{FVM}[7,8,14]$ and $\operatorname{FDM}[11]$ were furtherdeveloped to study the formation mechanisms of defects (e.g., porosityand balling).

The quantitative knowledge (e.g., temperature profiles) obtained using the molten pool models can be explored to study the solidification microstructure. Indeed, the thermal resultswereused for the microstructure calculation inAM modelsfor blown powder processes such as laser engineered net shaping (LENS)[15-18].It is noted that such microstructure calculation oftentimes used closed-form, empirical solidification equations. Recently, a thermodynamically consistent microstructure prediction for AMwas attempted based on thematerial composition and non-equilibrium solution[19].For powder bed processes, there is very limited research, which takes advantage of the thermal results calculated by the aforementioned molten pool AM models to studysolidification conditions and their effect onmicrostructure (e.g. solidification morphology and grain size).

Finally, it is noted that another important group of AM process models are based on numerical solution of heat conduction and stress equilibrium equations using finite element method (FEM). Although they are capable of describing transient temperature evolution, scanning path strategies and stress fields[20-23], the FEM based models typically use coarse mesh (e.g., mesh size on the order of 1 $\mathrm{mm}$ ) and are thus difficult in capturing the local melting and solidification conditions.

In thepresent paper, an existing 3-D transient molten pool physics model is extended to calculate the temperature field, fluid convection and molten pool shape in L-PBF of Inconel ${ }^{\circledR}$ Alloy 718 (IN718). The powder packing information is calculated from a DEM based packing model and then imported into the molten pool model as the initial geometry. From the calculated molten pool 
temperature field, the solidification parameters (i.e., temperature gradient and solidification rate)are computed. Those calculated solidification parametersare in turn used to quantitatively assess the solidification microstructure using existing modelsdeveloped for weld metals. The simulation results are compared to the experimental data measured on the IN718 coupon built by L-PBF.

\section{L-PBF Experiment}

A simple cubic coupon with dimensions of $15 \times 15 \times 15 \mathrm{~mm}^{3}$ was built on a flat IN718 substrate using an EOSINT M280 AM system. IN718 powder fabricated by gas atomization was used.Examination of the powder particles in scanning electron microscopy (SEM) revealed that the particles were fairly spherical in shape and had diameters varying from 20 to $40 \mu \mathrm{m}$. The M280 system hadan Ytterbiumfiber (Yb-fiber) laser with a maximum power of $400 \mathrm{~W}$. The actual laser power used for building the cube was $285 \mathrm{~W}$, and the laser travel speed was $0.96 \mathrm{~m} / \mathrm{s}$. The laser spot diameter was $100 \mu \mathrm{m}$. The nominal hatch spacing was about $116 \mu \mathrm{m}$. The height of each layer fused was $40 \mu \mathrm{m}$, and it took 375 layers to build to the height of $15 \mathrm{~mm}$. Argon was used as the shielding gas.

The as-built coupon was cross-sectioned at a center plane parallel to the building direction. The sectioned sample was prepared for metallographic observation of molten pool boundaries in optical microcopy and solidification microstructure in SEM.To help reveal the moltenpool boundaries, the sample was electrolytic etched in $10 \%$ oxalic for 5 to 10 seconds.

\section{Modeling Approaches}

The 3-D transient molten pool simulation was computationally expensive due to the fine mesh size and time increment used. The current simulationwas thus limited to laser fusion of a single layer of powder particles over an area of $1 \mathrm{~mm} \times 0.4 \mathrm{~mm}$ to keep the computation cost down to a reasonable level (e.g., less than 40 hours ofCPU clock-time). In spite of the small computational domain considered, important molten pool temperature data and solidification parameters were calculated,as discussed in the following.

\subsection{DEM simulationof powder bed structure}

An existing DEM based packing modelwas used to calculate the powder packing information for a single layer of IN718 powder particles laid on a solid block substrate. Details of this DEM based packing model is available in a recent paper by the authors[11].Several salient features are briefly summarized in the following.DEM is a well-established numerical method forsimulating the movement of a large number of discrete particles involving mechanical contactinteractions. Two essential simplifications are typically used by DEM[24]. First, the particles are approximated as solid spheres with predefined radii. Second,DEM utilizes the Hertz based soft-contact formula that permitsa small local overlap at the contact point between two particles, asillustrated in Fig. 1 . The normal contact force, $F_{n}$, is computed using the Hertz formula for the overlap distance. The tangential shear force, $F_{s}$, is calculated using Coulomb friction law.Finally, the gravitational force is addedwith the contact forces to obtain the total force and its moment exerting on each particle. 


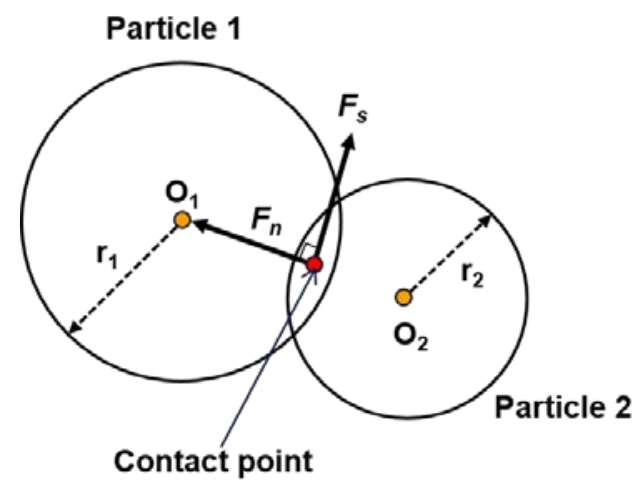

Figure 1.Contact forces generated by interaction between two spherical particles. $O_{i}$ and $r_{i}(i=1$ or 2$)$ are the center and radius of Sphere i, respectively.

Using an explicit time integration scheme, DEM solves the Newton's second law of motion to calculate the particle velocities (three translational and three rotational) at each time step. The position of a particle at the end of a time step are obtained by offsetting its position at the beginning of the step by the distance traveled and angle twisted (i.e.,multiplication of the respective velocities and time step size). The solution thenmarches to the next time step until the particles are eventually settled down.

The powder bed packing model was developed based on Yade, an open source DEM code [24].The mainmechanical properties used for DEM simulation included: Young's modulus $=195 \mathrm{GPa}$, and friction coefficient between particles $=0$.3. The size (diameter) distribution for the IN718 powderwas not available at the time of the present research. For simplicity, it was assumed that the particle size followed a positively skewed distribution based on the experimental measurement for a 316L stainless steel powder bySpieringset al.[6]. The powder diameter was set within 20 to $40 \mu \mathrm{m}$. The positively skewed distribution yielded high percentage of smaller particles (e.g., $90 \%$ of the particles having a diameter less than $28 \mu \mathrm{m})$.

The powder bed packing simulation consisted of the following steps. First, a cloud of about 10,000 spherical particles was generated right above a square powder container. The diameter of each particle was initiatedfrom the positively skewed distribution. Second, the particles dropped freely into the container. Third, mechanical vibration was imposed to the container to increase the packing density as the dropped particles in the second step were loosely packed. Fourth, a cylindrical roller was used to spread a $40-\mu \mathrm{m}$-high layer of the particles from the container to the powder bed. Finally, the coordinates and radii of individual particles on the powder bedwere exported.

It is noted that the cloud of particles was randomly generated in the first step. Although the particle size distribution followed the predefined distribution statistically, the exact diameters of individual particles varied from run to run. In addition, the initial position of a particle was also randomly assigned. Due to such randomness, the DEM results (e.g., surface topology) would not be identical for runs made with the same input parameters. To quantify the sensitivity of the surface topology calculated by DEM, three runs weremade. Results from each run were imported into the molten pool simulation to definethe initial geometry of powder packingon the substrate.

\subsection{Heat transfer and fluid flow simulation}


The existing molten pool model was developed based on Flow-3D, a commercial computational fluid dynamics (CFD) software[25]. The molten metalwas assumed to be Newtonian and incompressible.The model numerically solved the conservation equations of mass, momentum and energy, which are given in Eqns. (1) to (3), respectively:

Mass:

$$
\begin{gathered}
\nabla \cdot \vec{v}=0 \\
\frac{\partial \vec{v}}{\partial t}+(\vec{v} \cdot \nabla) \vec{v}=-\frac{1}{\rho} \nabla P+\mu \nabla^{2} \vec{v}+\vec{g}\left[1-\beta\left(T-T_{m}\right)\right] \\
\frac{\partial h}{\partial t}+(\vec{v} \cdot \nabla) h=\frac{1}{\rho}(\nabla \cdot \lambda \nabla T)
\end{gathered}
$$

where $\vec{v}$ is the velocity of molten material, $t$ is the time, $P$ is the pressure, $\mu$ is the viscosity, $g$ is the gravitational acceleration, $\beta$ is thethermal expansion coefficient, $T$ is the temperature, $T_{m}$ is the melting temperature of material, $h$ is the enthalpy, $\rho$ is the density, and $\lambda$ is the thermal conductivity. In Eqn. (3), the classic enthalpy-porosity method was used to capture the position of solid/liquid (S/L) interface of melting and solidification.Additional details about each term in Eqns. (1) to (3) are available in many literature publications $[7,11,12]$ and are thus not described here.

As discussed earlier, the location of the molten pool surface, a curved free surface, changes with time and needs calculated. The VOF method[25]was used to capture the position of the free surface (void-fluid interface) as a function of timeusing Eqn. (4):

Conservation of fluid volume fraction:

$$
\frac{\partial F}{\partial t}+\nabla \cdot(\vec{v} F)=0
$$

where $F$ is the fluid volume fraction $(0 \leq F \leq 1) . F=0$ indicates a void cell where no fluid is present, and $F=1$ indicates a liquid cell completely occupied by the fluid. $0<F<1$ indicates an interface cell containing the void-fluid interface. The VOF equation was solved concurrentlytogether with the conservation equations (1) to (3)to obtain the fluid volume fraction field. The fluid volume fractions of neighboring cells were then used to construct the interface position using the piecewise linear interface calculation (PLIC). Particularly, the normal vector of the interface for each interface cell was estimated based on the gradient of $F$. A planar surface with the calculated normal was constructed so that the inward part of the cell separated by the planar surface had the fluid volume for that particular cell. Finally, the boundary conditions including surface heat flux and surface tension forcewere updated based on the new interface position.

\subsection{Computational domain}

The 3-D computational domain had dimensions of $1000 \mu \mathrm{m}$ (length or X), $400 \mu \mathrm{m}$ (width or $\mathrm{Y}$ ) and $210 \mu \mathrm{m}$ (height or Z) as shown in Fig. 2. The domain consisted of a140- $\mu \mathrm{m}$-tall substrate, a $40-\mu \mathrm{m}$ tall powder layer, and a30- $\mu \mathrm{m}$-tall void region along the Z-direction. For the powder layer, the particle coordinates and radii calculated from the DEM packing model were imported and initiated as solid spheres with $F=1$. The substrate was also initiated as solid with $F=1$, whereas the void region was initiated as $F=0$.A biased mesh was used to attain finer mesh $(4 \mu \mathrm{m})$ around molten pool region and 
coarser mesh $(8 \mu \mathrm{m})$ in the substrate. Even for the small domain size considered, the use of refined mesh necessary for spatial resolution of individual powder particles yielded 1.25 million cells in total.

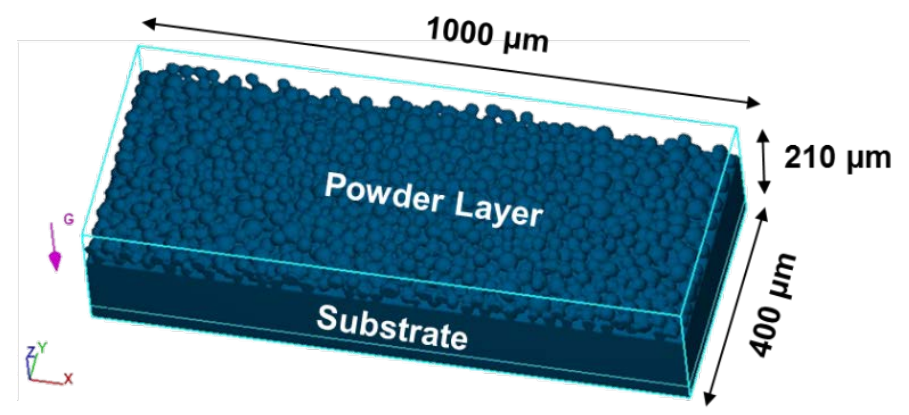

Figure 2. Computatoinal domain used for the 3-Dtransient heat and fluid flow simulation

The small computational domain considered, a necessity due to the high computational cost, limited the laser scanning pattern that could be simulated. In the present simulation, two overlapping tracks were considered, as shown in Fig. 3. The first laser track was made along thepositive X-direction. Then, the beamwas turned off andtranslatedin the negative Y-directionby $116 \mu \mathrm{m}$. After the beam was off for $120 \mu \mathrm{s}$, the beam was turn back on to make the second track along the negative X-direction. The laser scanning speed was $0.96 \mathrm{~m} / \mathrm{s}$, power $=285 \mathrm{~W}$ and hatch spacing $=116 \mu \mathrm{m}$. This pattern of two overlapping tracks could be thought to represent the corner region of an actual laser hatching. Such simple setup nevertheless allowedthe model to take into account the overlapping distance, which was shown to be an important parameter for surface quality and mechanical properties [26].

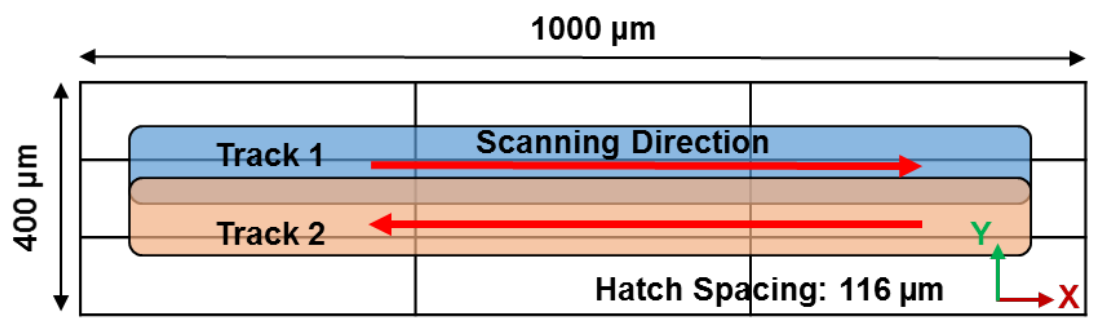

Figure 3. Laser scanning pattern oftwo overlapping tracks considered in the simulation

\subsection{Boundary conditions}

The thermal boundary conditions were needed to solve the energy conservation equation given by Eqn. (3). The heat input from the laser beam, imposed on the top free surface of powder layer, was approximated as a heat flux following a Gaussian distribution:

$$
q(w)=\frac{2 \eta Q}{\pi r^{2}} \exp \left(-\frac{2 w^{2}}{r^{2}}\right)
$$

where $q$ is the heat flux at a radial distancew from the beam center, $Q$ is the laser power, $r$ is the laser beam radius, and $\eta$ is theabsorptance of the material. In this study, a value of 0.38 was used for $\eta$ based on the range of absorptancevalues reported for powder bed $A M$ in the literature [13, 22].

The heat loss from the top free surface is described by the following equation: 


$$
q_{L}=h_{c}\left(T-T_{R}\right)-\sigma \varepsilon\left(T^{4}-T_{R}^{4}\right)+q_{\text {evap }}
$$

where $h_{c}$ is the convective heat transfer coefficient, $T$ is the surface temperature, $T_{R}$ is the room temperature, $\sigma$ is the Stefan-Boltzmann constant, and $\varepsilon$ is the emissivity.The first and second terms on the right hand side of Eqn. (6) correspond to the heat loss due to convection by inert shielding gas and radiation, respectively.

The last term of Eqn. (6) represents the heat loss by evaporation. Under the intense laser irradiation, the surface temperature of molten pool can reach the boiling temperature of material. As a result, the metal vapor forms and escapes the pool surface, carrying away a substantial amount of thermal energy.The evaporation cooling has a significant effect on the maximum temperature of molten pool, as reported in previous studies of AMand laser welding [7, 12].Assuming a homogenous mixture of metal vapor for simplicity, the evaporation heat loss is given as[7, 12]:

$$
q_{\text {evap }}=\frac{0.82 \Delta H_{v}^{*}}{\sqrt{2 \pi M R T}} P_{0} \exp \left(\frac{\Delta H_{v}^{*}\left(T-T_{v}\right)}{R T T_{v}}\right)
$$

where $\Delta H_{v}^{*}$ is the effective enthalpy of metal vapor, $M$ is the molar mass, $R$ is the universal gas constant, $T$ is the surface temperature, $P_{0}$ is the atmospheric pressure, and $T_{v}$ is the boiling temperature.

Due to the fast processing speed in L-PBF, it is expected that there is insufficient time for the prior fused layers to completely cool down before a new layer of powder is laid and fused. As discussed previously, the high computational cost limited the current model to simulating the laser fusing of a single layer; fusing of multiple layers was not considered.To account for the heat build-up during building, an effective preheating temperature was assigned as the initial temperature to the entire computational domain. An initial estimate of $573 \mathrm{~K}$ was used as this effective preheating temperature.

The main driving forces for fluid flow in the molten pool during L-PBF are described as follows. The first force is the Marangoni shear stress induced by the spatial variation of surface tension force:

$$
\gamma(T)=\gamma_{m}+\frac{d \gamma}{d T}\left(T-T_{m}\right)
$$

where $\gamma$ is the surface tension at the surface temperature $T, \gamma_{m}$ is the surface tension at the melting temperature, and $\frac{d \gamma}{d T}$ is the temperature coefficient of surface tension.

The second driving force is the recoil pressure due to theliquid metal evaporation, which exerts a normal force on the free surface[8]. Following a similar treatment for the evaporation heat loss, the recoil pressure, $P_{\text {recoil }}$, is given as $[7,8,12,27,28]$ :

$$
P_{\text {recoil }}=0.54 P_{0} \exp \left(\frac{\Delta H_{v}\left(T-T_{v}\right)}{R T T_{v}}\right)
$$

Temperature dependent material properties for IN718, including density, liquid metal viscosity, thermal conductivity and specific heat, are plotted in Fig. 4 [25].Values of the parameters needed to 
define the boundary conditions in Eqns. (5) to (9) are summarized in Table 1. The liquidus temperature was used as the melting temperature.

The 3-D transient simulation of laser fusing of two overlapping tracks took about 24 hoursof CPUclock time to complete in a workstation with Intel ${ }^{\circledR}$ Xeon ${ }^{\circledast}$ Processor E5335 and 4 GB RAM.
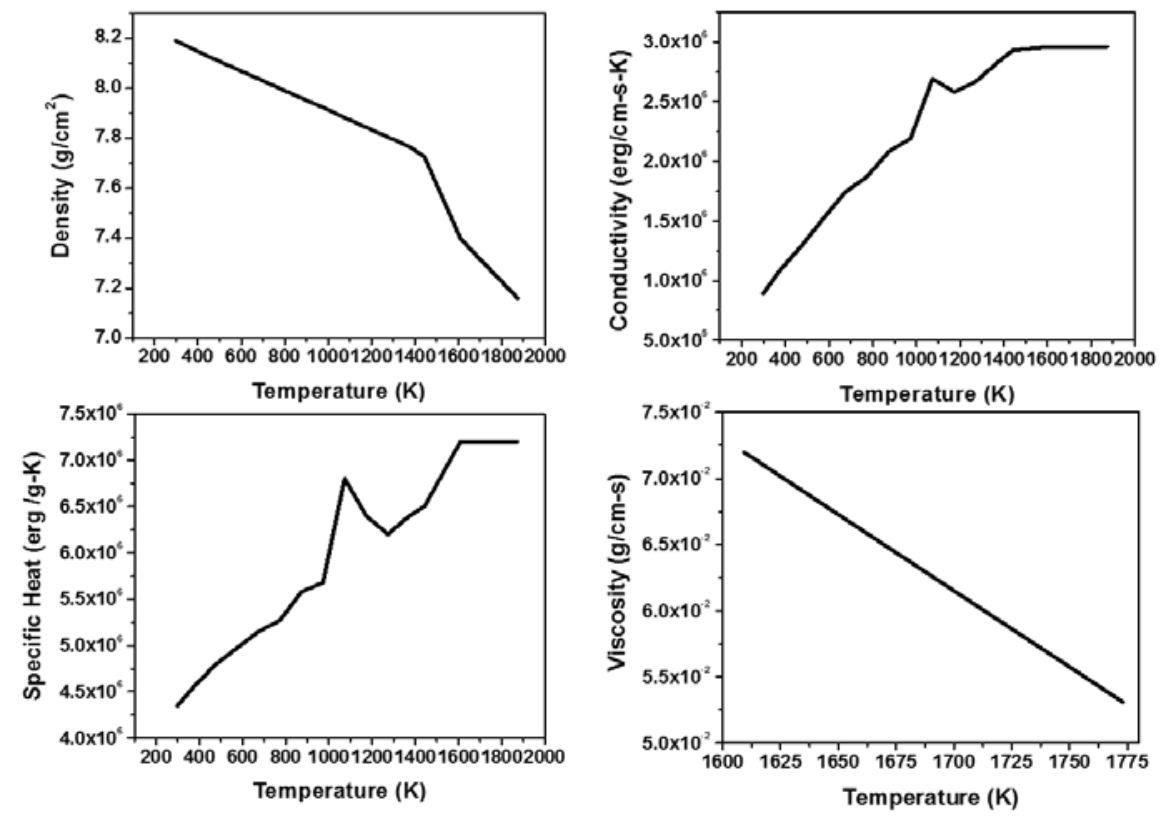

Figure 4.Temperature dependent thermo-physical properties of IN718. 1 erg $=10^{-7}$ joules.

Table 1. Processing parameters and material properties

\begin{tabular}{cc}
\hline Parameter and Property & Values (units) \\
\hline Laser scanning speed & $0.96(\mathrm{~m} / \mathrm{s})$ \\
\hline Laser power & $285(\mathrm{~W})$ \\
\hline Beam spot diameter & $100(\mu \mathrm{m})$ \\
\hline Solidus temperature & $1523.15(\mathrm{~K})$ \\
\hline Liquidus (melting) temperature & $1608.15(\mathrm{~K})$ \\
\hline Boiling temperature & $3188(\mathrm{~K})$ \\
\hline Surface tension at melting temperature & $1882\left(\mathrm{~g} / \mathrm{s}^{2}\right)$ \\
\hline Temperature coefficient of surface tension & $-0.1\left(\mathrm{~g} / \mathrm{s}^{2} \mathrm{~K}\right)$ \\
\hline Convective heat transfer coefficient & $80\left(\mathrm{~W} / \mathrm{m}^{2}\right)$ \\
\hline Emissivity & 0.8 \\
\hline Effective enthalpy of metal vapor & $6.4 \times 10^{10}(\mathrm{erg} / \mathrm{g})$ \\
\hline Molar mass of metal vapor & $59.75(\mathrm{~g} / \mathrm{mol})$ \\
\hline
\end{tabular}

\section{Results and Discussion}

As mentioned previously, three runs were made for the same parameters to quantify the sensitivity of the surface topology. A comparison of the simulation results from those three runs showed 
that there was a smalldifference in the calculated packing density (less than 1\%), molten pool dimensions(less than $3.2 \mu \mathrm{m}$ ), and peak temperatures(less than 20K). The sensitivity of the surface topology seems negligible for the conditions considered in the present study. Hence, the simulation results for one caseare discussed in details as follows.

\subsection{Temperature and velocity fields}

A 3-D view of the computed temperature field at the end of fusing the first and second tracksis shownin Fig. 5(a) and (b), respectively. The velocity vector field in the molten pool is not drawn for clarity of visualization. The computational domain is sectioned along the center $\mathrm{X}-\mathrm{Z}$ plane of each track in order to show the through-thickness temperature profile. The molten pool is plotted as the red region, which temperature is at and above the liquidus temperature (1608.15K).Partially-melted particles are observed around the periphery of the molten pool, and un-melted particles are located further away from the pool.The peak temperatures at the end of first and second tracks are calculated to be $3283 \mathrm{~K}$ and $3240 \mathrm{~K}$, respectively.

As shown in Figs. 5(a) and (b), the melt pool is elongated due to high scanning speed of $0.96 \mathrm{~m} / \mathrm{s}$, as expected. It is observed the molten pool surface is significantly depressed underneath the laser beam. This is due to the evaporation recoil pressure exerting on the molten pool surface as the molten metal is heated above the boiling temperature $(3188 \mathrm{~K}$ ) by the intense laser heat input. The molten pool for the second track is noticeably wider than that for the first track, as discussed in more details later.

The molten pool convection on the center X-Z plane is plotted in Figs. 5(c) and (d) at the end of fusing the first and second tracks, respectively. The velocity field is visualized as arrows, which lengths indicate their magnitudes. As described previously, the molten pool is significantly depressed directly underneath the laser beam, resulting in a downward flow within the thin layer of molten pool there. Right behind the laser, a strong backward surface flow (toward the tail of the pool) is formed driven by the Marangoni shear stress. Further behind the laser, the top surface profile is relatively flat and the surface temperature gradient is relatively low. As a result, the convection is more stagnantin the tail portion of the pool. The convective heat transfer is a dominant mechanism for heat transport within the molten pool. The fluid convection significantly influences the pool shape (e.g., the free surface and penetration profiles) and subsequently the solidification conditions.

The molten pool profile for the first and second tracks is more clearly seen in Figs. 6(a) and (b), respectively. These figures plot the calculated temperature field on the $\mathrm{Y}-\mathrm{Z}$ plane (perpendicular to the travel direction) at the half-length location. The snapshot of temperature field is taken at time $=540 \mu \mathrm{s}$ for the first track and at time $=1510 \mu \mathrm{s}$ for the second track, when the melt pool reaches its maximum size. The initial height of powder layer is about $40 \mu \mathrm{m}$. The height of the molten pool, resulting from fusing the powder layer, is only $24 \mu \mathrm{m}$. Such "shrinkage" of fused layer is expected as the packed powder is only 40 to $50 \%$ dense and contains a significant void volume.

As shown in Fig. 6(a), the melt pool shape for the first track is relatively symmetric about the laser center. On the other hand, an asymmetric molten pool for the second track is observed in Fig. 6(b), where the molten pool is widened to the right toward the first track. The different molten pool shapes can be better explained by comparing the transverse temperature profiles at the original substrate surface, marked as black dashed lines in Figs. 6(a) and (b). These transverse temperature profiles are plotted in Figs. 6(c) and (d) for the first and second tracks, respectively. 

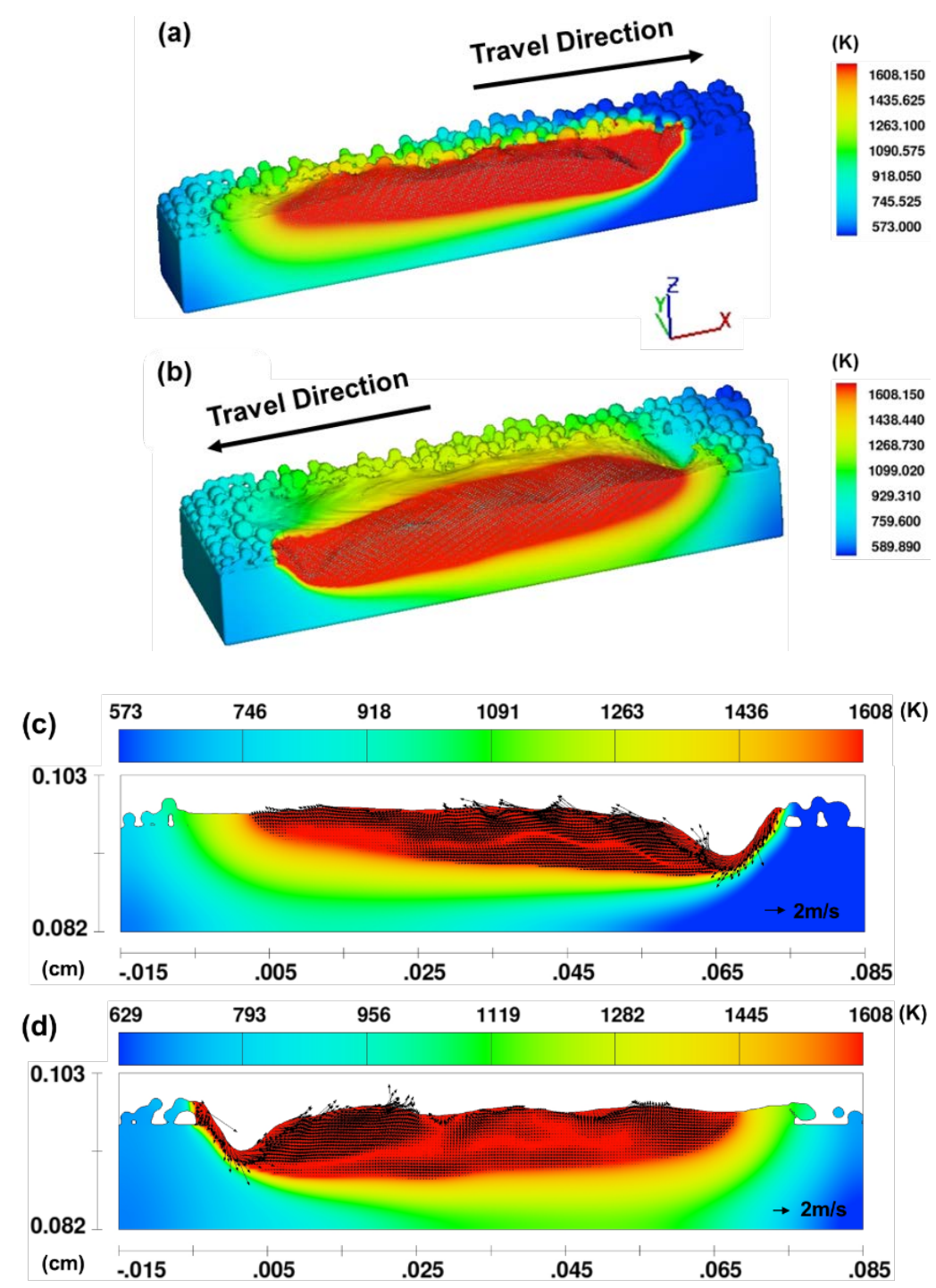

Figure 5. 3-D longitudianl section view of temperature fields: (a) and (b), and 2-D view of velocity fields: (c) and (d). (a) and (c) are plottedat the end of fusingthefirst track, and (b) and (d) for the second track.

Figure $6(\mathrm{c})$ shows the temperature profiles for the first track at two times. For both times, the temperature profile is symmetric about the laser center positioned at $Y=-0.4 \mu \mathrm{m}$ with the peak temperature located at the center. The symmetric temperature profile for the first track is expected as the heat is evenly conducted through the powder layer and substrate.For the second track, the laser center is positioned at $\mathrm{Y}=-11.6 \mu \mathrm{m}$. At $\mathrm{t}=1290 \mu \mathrm{s}$, the laser center just passes the particular $\mathrm{Y}-\mathrm{Z}$ monitoring plane. At this time, the temperatures within the molten pool (i.e., above $1608.15 \mathrm{~K}$ ) are still relatively symmetric with the peak temperature located at the center, as shown in Fig. 6(d). On the other hand, the temperature in the substrate equidistant to the laser center is higher in the right region toward the first track. This is because the region around the first track only cools down to about $1100 \mathrm{~K}$ at the time of depositing the second track. The temperature profile becomes more asymmetric at $\mathrm{t}=$ 
$1510 \mu \mathrm{s}$. The significant "preheating" from the first track helps the molten pool for the second track expand more toward the right with the peak temperature also shifted to the right of the laser center.
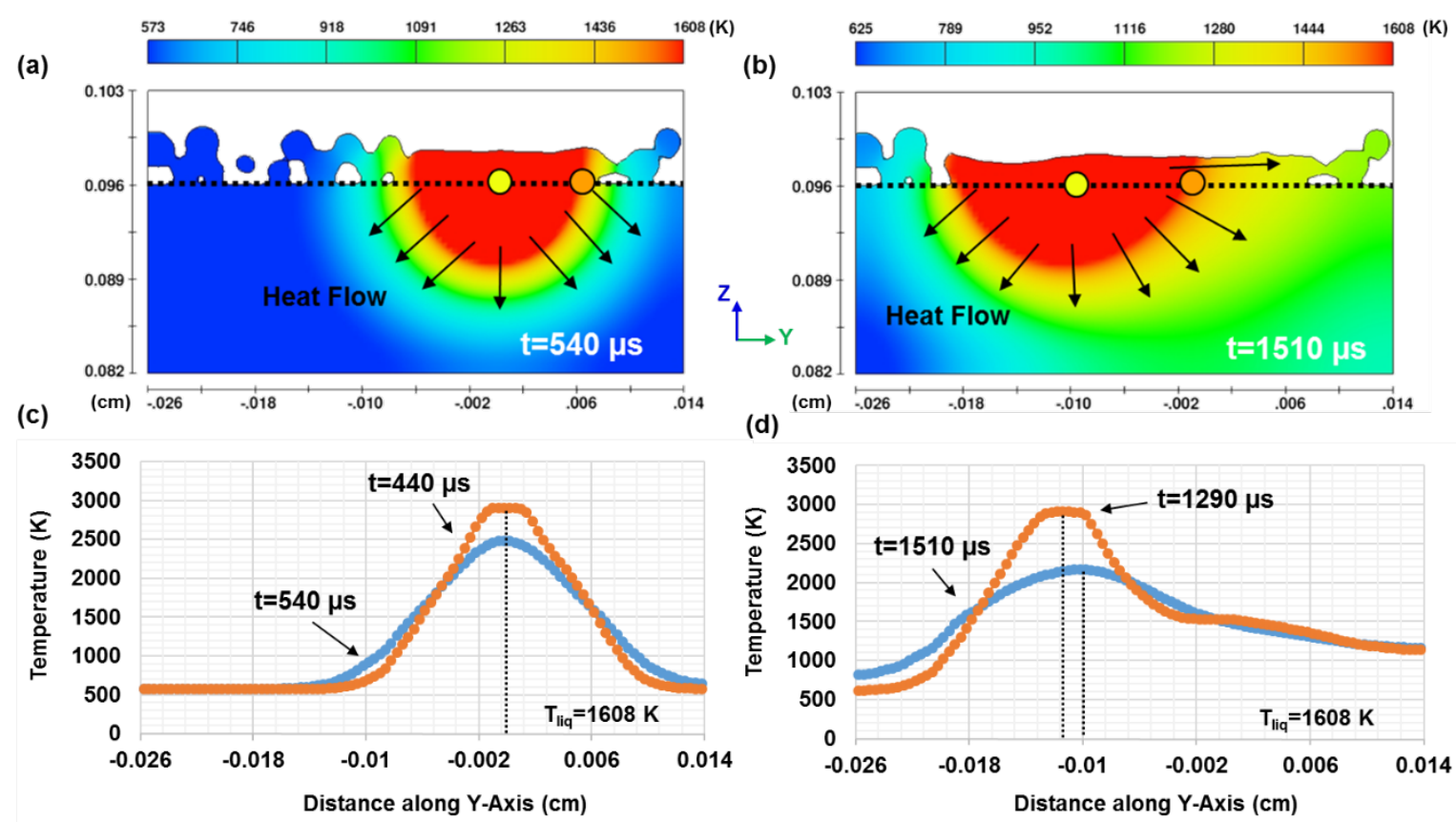

Figure6. Temperature field at (a)540 $\mu$ sfor the first track,and(b) $1290 \mu$ s for the second track. (c) and (d) plot the transverse temperature profile at two times for the first and second tracks, respectively.

It is noted that another factor contributing to the asymmetric temperature field for the second track is the powder versus solid material surrounding the molten pool. In other words, the first track is surrounded by powder particles on both left and right sides during solidification, as shown in Fig. 6(a). On the other hand, the powder particles are present only on the left side during solidification of the second track. The right side of the second track is surrounded by the solid layer fused in the first track, as shown in Fig. $6(\mathrm{~b})$. The packed powder has a lower ability to conduct heat compared to the solid, and such difference in thermal conductivity can influencethe temperature distribution [23].

To more clearly illustrate the transients of molten pool (melting and solidification), the calculated temperature and fluid convection fields on a Y-Z monitoring plane for the first track is plotted as a function of timein Fig. 7. The laser beam travels along the $X$ direction, i.e., coming out of the page.Figure 7(a) shows the initial geometry, which is defined based on the DEM simulation results. At $\mathrm{t}=$ $90 \mu \mathrm{s}$, the particles, just irradiated by the laser beam,start tomelt and coalesce into the molten pool, as shown in Fig. 7(b). As the beam moves closer to the monitoring plane, the substrate also melts, resulting in widening and deepening of the molten pool, as shown in Figs. 7(c) and (d). The molten pool surface in those two figures is noticeably depresseddue to the recoil pressure fromevaporation.Figure 7(e) shows the trailing section of the molten pool after the beam passes the monitoring plane. Solidification starts as the beam moves further away from the plane, as shown in Figs. 7(g) to (i). The entire cross section is solidified very quickly within $790 \mu \mathrm{s}$ (from $\mathrm{t}=240$ to $1030 \mu \mathrm{s}$ ). It is interesting to observe the temperature field is asymmetric about the laser beam center especially during solidification. Such 
asymmetry indicates that the local powder configuration adjacent to the molten pool can have a marked effect on the temperature distribution and solidification conditions.

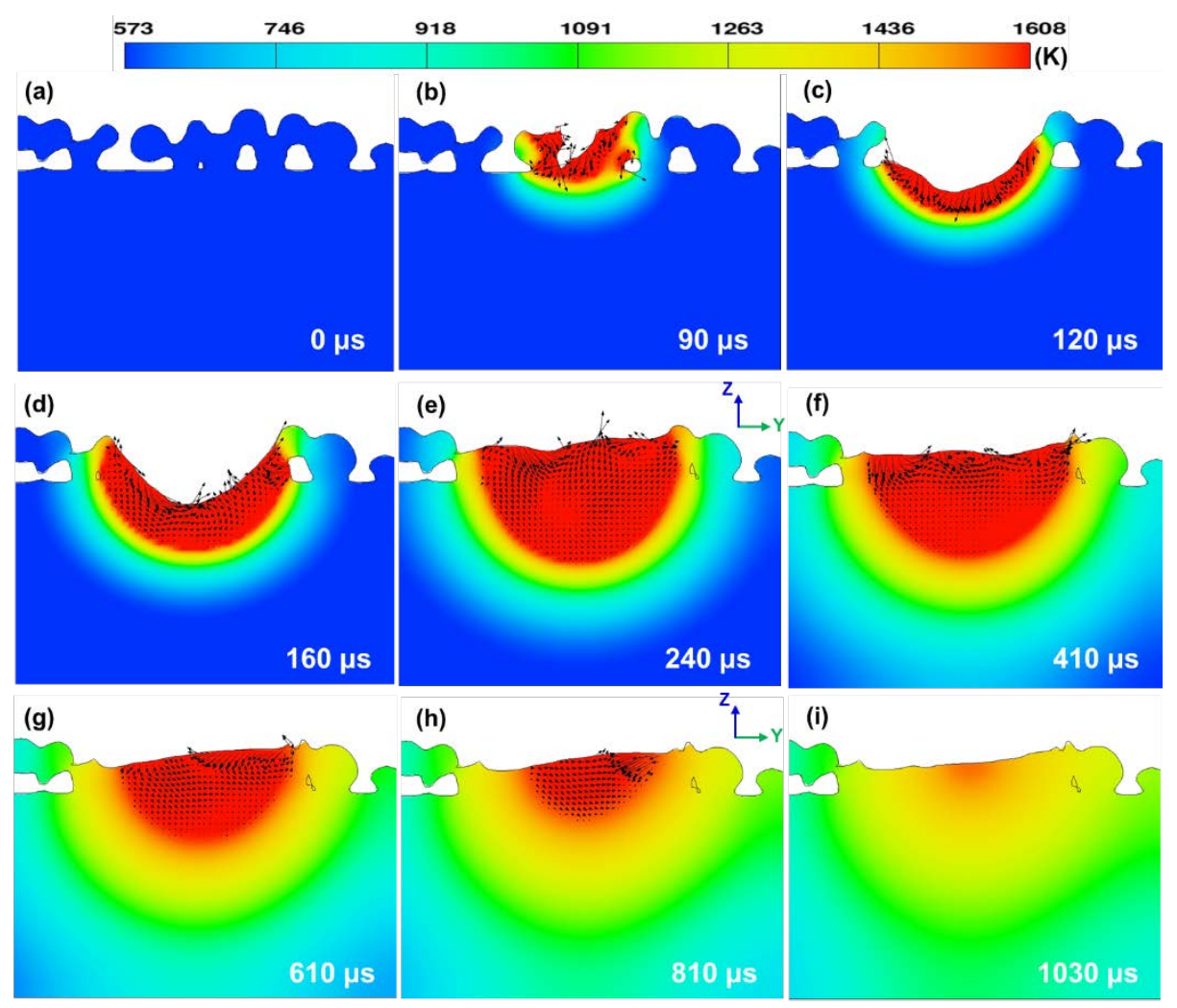

Figure 7. Evolution of molten pool heat transfer and fluid convection at a fixed $\mathrm{Y}-\mathrm{Z}$ cross sectionforthe melting and solidification of the first track.

\subsection{Model validationof molten pool boundaries}

The $\mathrm{Y}-\mathrm{Z}$ cross section of the molten pool at its maximum size for the first and second tracks is plotted in Fig. 8(a), where the blue dashed lines highlight the molten pool boundaries. Figure 8(b) shows a small area on the cross section from the actual IN718 coupon, highlighting two overlapping tracks. On the actual coupon, there was a significant extent of re-melting between layers. Moreover, the molten pool boundaries on the cross section were oftentimes not those perpendicular to the laser travel direction because of the complex laser hatching in building the IN718 coupon. After observing the molten pool profiles at over 50 different locations in the coupon, the small area in Fig. 8(b) is identified to contain the most "intact" boundaries of the molten pool on the observed cross section perpendicular to the laser travel direction.

As shown in Fig. 8, the shape of molten pool calculated using the heat transfer and fluid flow model is comparable to the experimental measurement. Particularly, the calculated asymmetric shape of the second track, widening toward the first track, is consistent with that observed experimentally. The calculated versus measured dimensions of the molten pool are summarized in Table 2 . The error 
between the calculated and measured molten pool dimensions is relatively small, indicating the validity of the temperture field calculated using the molten pool model.

(a)

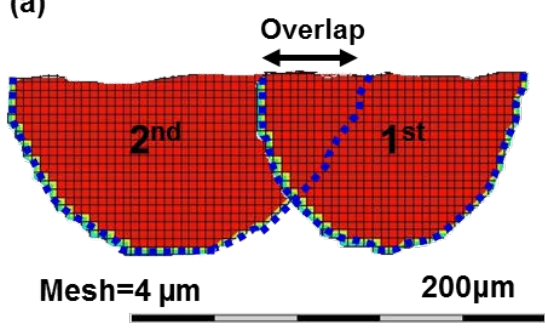

(b)

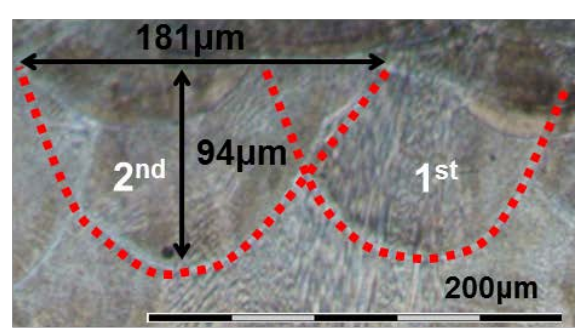

Figure 8. Comparisoin of (a) calculated and (b) experimentally measured molten pool shapes

Table 2. Comparison of calculated and measured molten pool dimensions

\begin{tabular}{ccccc}
\hline Track no. & Dimension & Experiment & Modeling & Error \\
\hline \multirow{2}{*}{1} & Width & $144 \mu \mathrm{m}$ & $132 \mu \mathrm{m}$ & $9.1 \%$ \\
& Depth & $90 \mu \mathrm{m}$ & $84 \mu \mathrm{m}$ & $7.1 \%$ \\
\hline \multirow{2}{*}{2} & Width & $181 \mu \mathrm{m}$ & $176 \mu \mathrm{m}$ & $2.8 \%$ \\
& Depth & $94 \mu \mathrm{m}$ & $86 \mu \mathrm{m}$ & $9.3 \%$ \\
\hline \multirow{2}{*}{$1-2$} & Overlap & $63 \mu \mathrm{m}$ & $56 \mu \mathrm{m}$ & $12.5 \%$ \\
\hline
\end{tabular}

\subsection{Solidification morphology}

The calculated temperature field, such as that shown in Fig. 5, can be used to directly calculate two important solidification parameters: temperature gradient and solidification rate. Individual components of temperature gradient, $\vec{G}_{x}, \vec{G}_{y}$ and $\vec{G}_{z}$, are obtained by differentiating the temperature profile at the $\mathrm{S} / \mathrm{L}$ interface of the molten pool with respect to the $\mathrm{x}, \mathrm{y}$ and $\mathrm{z}$ coordinates, respectively. The temperature gradient normal to $S / L$ interface, $\vec{G}_{n}$, isthen calculated using the simple expression below:

$$
\left|\vec{G}_{n}\right|=\sqrt{\vec{G}_{x}^{2}+\vec{G}_{y}^{2}+\vec{G}_{z}^{2}}
$$

The solidification rate, $\vec{R}_{S}$, normal to the $\mathrm{S} / \mathrm{L}$ interface,can be calculated based on the following geometrical relationship $[18,29]$ :

$$
\left|\vec{R}_{s}\right|=v \cdot \cos \theta=v \cdot \frac{\left|\vec{G}_{x}\right|}{\left|\vec{G}_{n}\right|}
$$

where $v$ is the travelspeed of laser beam whichmovesalong the $\mathrm{X}$ direction, and $\theta$ is the angle between the normal direction of local $\mathrm{S} / \mathrm{L}$ interface and the laser travel direction (i.e., the $\mathrm{X}$ direction).

The solidification morphology can be evaluated usingthe ratio of $\vec{G}$ to $\vec{R}[30]$. A decrease in the $\vec{G} / \vec{R}$ ratio corresponds toa transition of solidification morphology from planar to cellular, columnar dendrite and eventuallyequiaxedgrains. To evaluate the solidification morphology in the molten pool 
during L-PBF, the solidification parameters at the molten pool side edge ("edge") and its trailing end ("tail") are calculated.These two monitoring locations are schematically shown inFigs. 9(a) and (b).

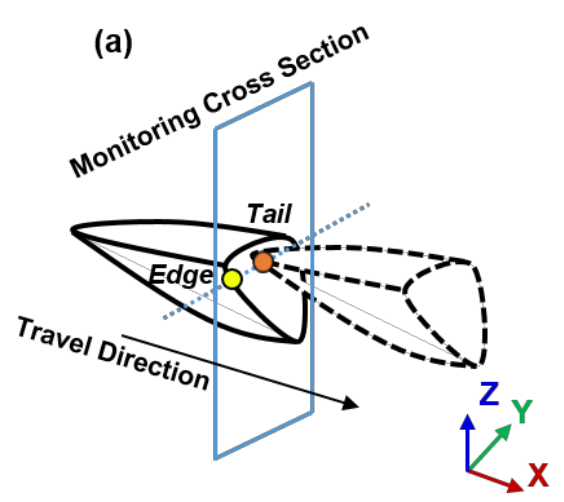

(b) Monitoring Cross Section

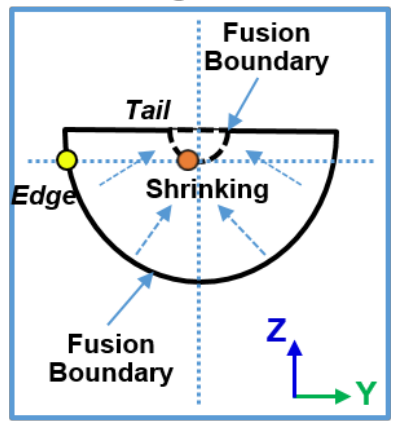

Figure 9. Schematics showing the edge and tail monitoring locations selected to analyze the solidification conditions: (a) 3D view, and (2) 2D cross-section view

In general, the temperature gradient $\vec{G}$ is high at the melt pool side edge wherethe solidification rate $\vec{R}$ is slow [30]. On the other hand, at the molten pool trailing end, the solidification rate is fast (reaching the laser scanning speed) whereas the temperature gradient is small. In other words, those two locations correspond to the minimums and maximums of solidification parameters. Table 3 summarizes the calculated $\vec{G}_{n}$ and $\vec{R}_{s}$ at thosetwo monitoring locations for the first and second tracks.

Table 3. Caclulated $\vec{G}_{n}$ and $\vec{R}_{S}$ at the side and trailing edges of the molten pool for the $1^{\text {st }}$ and $2^{\text {nd }}$ tracks

\begin{tabular}{rccc}
\hline \multicolumn{2}{c}{ Location } & Temperature Gradient $\left(\overrightarrow{\boldsymbol{G}}_{\boldsymbol{n}}\right)$ & Solidification Rate $\left.\overrightarrow{(\overrightarrow{\boldsymbol{R}}}_{\boldsymbol{s}}\right)$ \\
\hline \multirow{2}{*}{$1^{\text {st }}$ track } & Edge & $1.84 \times 10^{7}(\mathrm{~K} / \mathrm{m})$ & $0.05(\mathrm{~m} / \mathrm{s})$ \\
\cline { 2 - 4 } & Tail & $2.93 \times 10^{6}(\mathrm{~K} / \mathrm{m})$ & $0.70(\mathrm{~m} / \mathrm{s})$ \\
\hline \multirow{2}{*}{$2^{\text {nd }}$ track } & Edge & $7.88 \times 10^{6}(\mathrm{~K} / \mathrm{m})$ & $0.09(\mathrm{~m} / \mathrm{s})$ \\
\cline { 2 - 4 } & Tail & $3.42 \times 10^{6}(\mathrm{~K} / \mathrm{m})$ & $0.71(\mathrm{~m} / \mathrm{s})$ \\
\hline
\end{tabular}

The calculated values of $\vec{G}_{n}$ and $\vec{R}_{S}$ for the L-PBF molten pool are superimposed in an existing solidification process map of IN718 in Fig. 10. This map was calculated usinganalytical models developed originally for laser welding and cladding[31,32] and wasshown also to be applicable toassess the solidification morphology of IN718 made by electron beam melting (EBM), anotherpowder bed AM process[33]. As shown in Fig. 10, all four pointsin Table 3 lay outside the columnar/mixed transition line, indicating that the columnar dendrite is the dominant morphology in IN718 made by L-PBF.

SEM images of as-built solidification microstructure are shown in Fig. 11(a), where very fine grains are observed. A further analysis using electron backscatter diffraction (EBSD) shows much larger microstructure regions and thus does not suggest those small grains are equiaxed, as shown in Fig. 11(b).In other words, the solidification microstructure likely consists a mixture of predominantly columnar dendrite with some cellular.Such experimental observation is consistent with the solidified microstructure predicted based on the process map in Fig. 10. The dendrite size of the columnar 
microstructure is also assessed using another combined form of $\vec{G}_{n}$ and $\vec{R}_{s}$, as discussed in the next section.

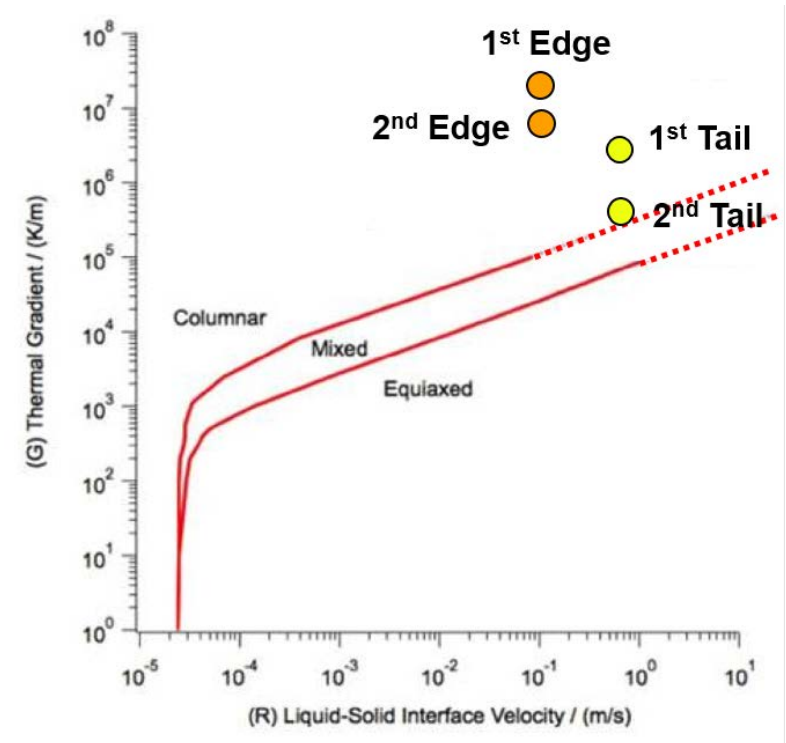

Figure 10. Calculated $\vec{G}_{n}$ and $\vec{R}_{S}$ plotted on a solidification process map for IN718[33]
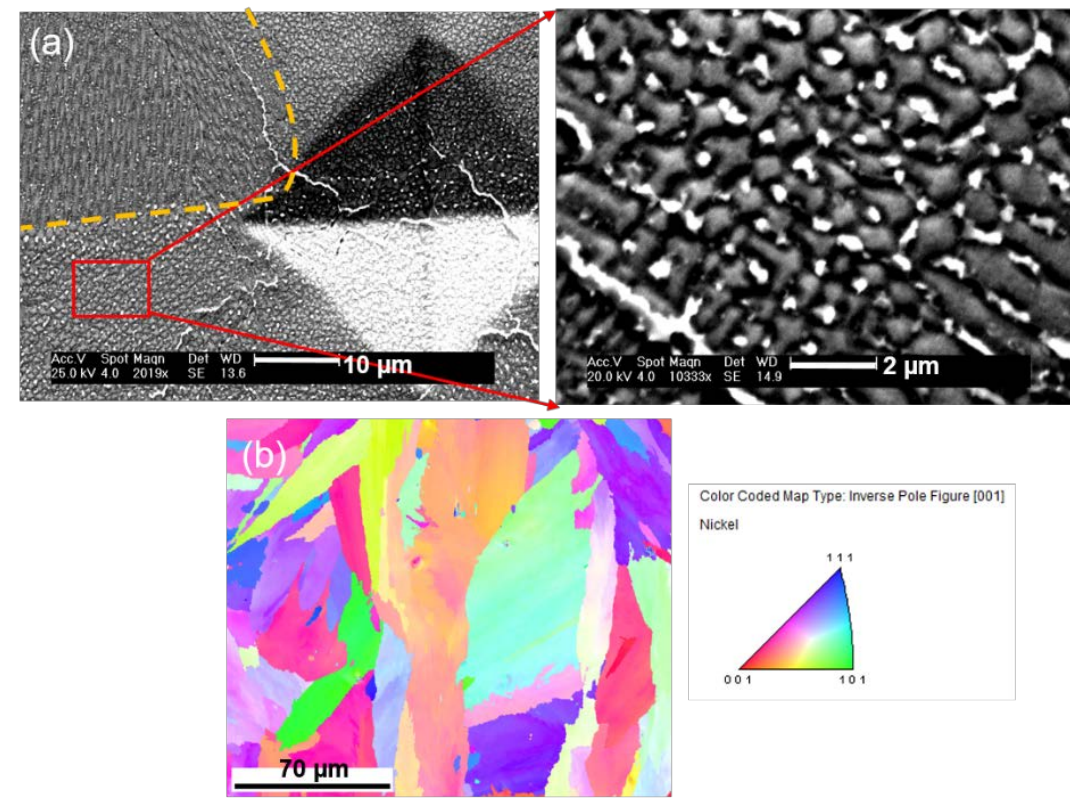

Color Coded Map Type: Inverse Pole Figure [001]

Nickel

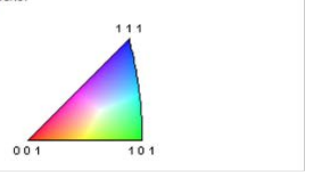

Figure 11. (a) SEM images of as-built solidification microstructure showing a mixture of predominantly columnar dendrite with some cellular. The right image in (a) is a magnified view of the red rectangular region in the left image. (b) EBSD image showing the grain texture.

\subsection{Dendritesize}


The primary dendrite arm spacing (PDAS) is an important microstructure parameter as a decrease in PDAS (and thus finer dendrite) generallyimproves the strength of as-built material.As discussed previously, due to the rapid solidification, the solidification microstructure appears as a mixture of predominantly columnar dendrite with some cellular. The growth of secondary dendrite arms is significantly suppressed, making it difficult to experimentally characterize the secondary dendrite arm spacing. Such fine microstructure produced in additive manufacturing was also reported in the literature $[34,35]$

The relationship between solidification parameters and PDASwasestablishedforlaser welding, hybrid laser-arc welding and blown powder type of AM of Ni-base superalloys[36-38]. The general formula for calculating PDAS, $\lambda$, is given by the following expressions[39-41]:

$$
\begin{aligned}
& \lambda=A \cdot G^{-a} \cdot R^{-b} \\
& A=4.3 \Delta T_{n}^{0.5}\left(\frac{D_{L} \Gamma}{\Delta T_{0} k}\right)^{0.25} \\
& A=2.83\left(L \Delta T_{0} k D_{L} \Gamma\right)^{0.25}
\end{aligned}
$$

where $A$ is a material dependent coefficient which can be calculated by two empirical equations: KurzFisher and Trivedi equations given in Eqns. (13) and (14), respectively. Physical properties of IN718 needed for calculating $A$ are summarized in Table 4. For the exponentsin Eqn. (12), $a=0.5$ and $b=0.37$ based on the values available in the literature[42].

Table 4. Physical properties of IN718 for PDAS calculation $[41,43]$

\begin{tabular}{cc}
\hline Physical Property & Value (units) \\
\hline Non-equilibrium solidification range $\left(\Delta T_{n}\right)$ & $140(\mathrm{~K})$ \\
\hline Diffusion coefficient in liquid $\left(D_{L}\right)$ & $3.0 \times 10^{-9}\left(\mathrm{~m}^{2} / \mathrm{s}\right)$ \\
\hline Gibbs-Thomson coefficient $(\Gamma)$ & $1.0 \times 10^{-7}(\mathrm{~K} \cdot \mathrm{m})$ \\
\hline Equilibrium solidification range $\left(\Delta T_{0}\right)$ & $85(\mathrm{~K})$ \\
\hline Partition coefficient $(k)$ & 0.48 \\
\hline Constant of harmonic perturbation $(L)$ & 28 \\
\hline
\end{tabular}

Table 5 summarizes the PDAS calculated for the $G$ and $R$ values in Table 3. The calculated PDAS is 1.32-1.87 $\mu \mathrm{m}$ for Trivedi equation and 1.57-2.23 $\mu \mathrm{m}$ for Kurz-Fisher equation. As shown in Fig. 11, the solidification microstructure consists of columnar dendrites (dark colored) and precipitates (light colored) located in the inter-dendritic regions. The measured value of PDAS is 1.0-1.8 $\mu \mathrm{m}$. The calculated PDAS is consistent with that measured experimentally on the actual coupon. For the solidification parameters considered here, the Trivedi equation yields PDAS slightly closer to the measured value than Kurz-Fisher equation.

Table 5. Calculated PDAS for the first and second tracks 


\begin{tabular}{cccc}
\hline \multirow{2}{*}{ Location } & \multicolumn{2}{c}{ PDAS } \\
\cline { 2 - 4 } & & Trivedi & Kurz-Fisher \\
\hline \multirow{2}{*}{$1^{\text {st }}$ track } & Edge & $1.55(\mu \mathrm{m})$ & $1.84(\mu \mathrm{m})$ \\
\cline { 2 - 4 } & Tail & $1.44(\mu \mathrm{m})$ & $1.71(\mu \mathrm{m})$ \\
\hline \multirow{2}{*}{$2^{\text {nd }}$ track } & Edge & $1.87(\mu \mathrm{m})$ & $2.23(\mu \mathrm{m})$ \\
\cline { 2 - 4 } & Tail & $1.32(\mu \mathrm{m})$ & $1.57(\mu \mathrm{m})$ \\
\hline
\end{tabular}

\section{Summary and Conclusions}

In summary, a computational framework with meso-scalespatial resolution is developed for simulation of solidification microstructure inL-PBF of IN718. The framework makes use of two main models in sequence. A DEM based packing model is used to calculate the powder packing information, which is subsequently imported to the molten pool model as the initial geometry. The 3-D transient molten pool model calculates the heat transfer, fluid flow and free surface evolution by solving the conservation equations based on the VOF method. A side-by-side laser scan consisting of two overlapping tracks are simulated. The temperature field is then used to compute the solidification parameters, which in turn, are used to assess the solidification microstructure including solidification morphology and dendrite size.

From the analysis of simulation results, the following conclusions can be drawn:

- An elongated molten pool along the travel direction is formed due to the fast scanning speed of the laser. Underneath the laser, the top surface of the molten pool is significantly depressed due to the evaporation recoil pressure. The depressed pool surface and the downward fluid flow below the laser are responsible for forming the penetration profile. Right behind the laser, there exists a backward surface flow driven by the spatial gradient of surface tension.

- The calculated transverse section profile of the molten pool is found to be relatively symmetric about the laser center for the first track. On the other hand, the pool shape for the second track is asymmetric, with the pool widened toward the side of the first track. This is primarily due to the pre-heat from fusing the first track. The predicted pool boundaries (including the asymmetric shape) is consistent with that measured experimentally on the actual IN718 coupon.

- Using the calculated temperature gradient $G$ and solidification rate $R$, it is assessed the solidification microstructure for L-PBF of IN718. The solidification morphology is evaluated to be predominantly columnar with some cellular, and the dendrite size (PDAS) is estimated to be 1.32-1.87 $\mu \mathrm{m}$. Such fine dendritic microstructure is formed due to the high values of both $G$ and $R$ in the molten pool. The calculated solidification morphology and PDAS are consistent with the experimentally observed morphology and size, respectively. The good agreement between the modeling and experimental results indicates that the solidification theories and models developed originally for welding processes can be effectively extended to the L-PBF process involving high $G$ and $R$ and thus high cooling rates.

Due to the high computational cost, the 3-D transient heat transfer and fluid flow simulation in the present study is limited to a simple side-by-side laser scan on a single layer. The complex laser 
hatching pattern used in the actual build makes accurately extracting the pool boundaries for model validation difficult. Nevertheless, the 3-D model, capturing essential characteristics of laser powder bed AM (processing parameters, powder packing, and free surface flow),computes the molten pool boundaries and solidification microstructure that are consistent with the experimental data. Such quantitative knowledge is essential to further understand and eventually controlthe local solidification microstructure in L-PBF additive manufacturing.

With the aid of high performance computingin the future, theframework will be expanded to simulate more complex scanning patterns and multiple layer deposition.Such a scaled-up modelhas the potentialto study the defect formation in the molten pool and to provide accurate temperature profiles to a FEM based model for predicting residual stresses in the built part.

\section{Acknowledgements}

The authors would like to acknowledgea grant from Office of Naval Research (ONR), Award No. N00014-14-1-0688, in support of the research.The authors thank Dr. Shawn Kelly of Edison Welding Institute (in Columbus, Ohio, USA) for performing the laser-powder bed fusion of IN718 coupon, and Ms. Hye-Yun Song of The Ohio State University for providing the experimental characterization data.

\section{References}

[1] S.S. Babu and R. Goodridge, Additive manufacturing, Mater. Sci. Technol., 31 (2015) 881-883.

[2] B. Schoinochoritis, D. Chantzis and K. Salonitis, Simulation of metallic powder bed additive manufacturing processes with the finite element method: A critical review, Proc IMechE Part B: J Eng Manuf, (2015) 0954405414567522.

[3] W.J. Sames, K.A. Unocic, R.R. Dehoff, T. Lolla and S.S. Babu, Thermal effects on microstructural heterogeneity of Inconel 718 materials fabricated by electron beam melting, J. Mater. Res., 29 (2014) 1920-1930.

[4] A. Raghavan, H.L. Wei, T.A. Palmer and T. DebRoy, Heat transfer and fluid flow in additive manufacturing, J. Laser. Appl., 25 (2013) 052006.

[5] B. Liu, R. Wildman, C. Tuck, I. Ashcroft and R. Hague, Investigation the effect of particle size distribution on processing parameters optimisation in Selective Laser Melting process, in: Proc. 26th Solid Free. Fabr. Symp., Austin, USA, 2011, pp. 227-238.

[6] A.B. Spierings, N. Herres and G. Levy, Influence of the particle size distribution on surface quality and mechanical properties in AM steel parts, Rapid Prototyping J., 17 (2011) 195-202.

[7] F.J. Gurtler, M. Karg, K.H. Leitz and M. Schmidt, Simulation of laser beam melting of steel powders using the three-dimensional volume of fluid method, Phys. Proced., 41 (2013) 874-879.

[8] C. Qiu, C. Panwisawas, M. Ward, H.C. Basoalto, J.W. Brooks and M.M. Attallah, On the role of melt flow into the surface structure and porosity development during selective laser melting, Acta Mater., 96 (2015) 72-79.

[9] C. Körner, E. Attar and P. Heinl, Mesoscopic simulation of selective beam melting processes, J. Mater. Process. Technol., 211 (2011) 978-987.

[10] I. Kovaleva, O. Kovalev and I. Smurov, Model of heat and mass transfer in random packing layer of powder particles in selective laser melting, Phys Procedia, 56 (2014) 400-410.

[11] Y.S. Lee and W. Zhang, Mesoscopic simulation of heat transfer and fluid flow in laser powder bed additive manufacturing, in: Proc. 26th Solid Free. Fabr. Symp., Austin, USA, 2015, pp. 1154-1165. 
[12] C. Jung-Ho, F.F. Dave, O.M. John and J.H. Kendall, Weld pool flows during initial stages of keyhole formation in laser welding, J. Phys. D: Appl. Phys., 42 (2009) 175502.

[13] S.A. Khairallah and A. Anderson, Mesoscopic simulation model of selective laser melting of stainless steel powder, J. Mater. Process. Technol., 214 (2014) 2627-2636.

[14] W. King, A.T. Anderson, R.M. Ferencz, N.E. Hodge, C. Kamath and S.A. Khairallah, Overview of modelling and simulation of metal powder bed fusion process at Lawrence Livermore National Laboratory, Mater. Sci. Technol., 31 (2015) 957-968.

[15] V.D. Manvatkar, A.A. Gokhale, G. Jagan Reddy, A. Venkataramana and A. De, Estimation of melt pool dimensions, thermal cycle, and hardness distribution in the laser-engineered net shaping process of austenitic stainless steel, Metall. Mater. Trans. A, 42 (2011) 4080-4087.

[16] V. Manvatkar, A. De and T. DebRoy, Heat transfer and material flow during laser assisted multi-layer additive manufacturing, J. Appl. Phys., 116 (2014) 124905.

[17] S.M. Thompson, L. Bian, N. Shamsaei and A. Yadollahi, An overview of direct laser deposition for additive manufacturing; Part I: Transport phenomena, modeling and diagnostics, Addit. Manuf., 8 (2015) 36-62.

[18] Z. Liu and H. Qi, Effects of processing parameters on crystal growth and microstructure formation in laser powder deposition of single-crystal superalloy, J. Mater. Process. Technol., 216 (2015) 19-27.

[19] J. Smith, W. Xiong, J. Cao and W.K. Liu, Thermodynamically consistent microstructure prediction of additively manufactured materials, Comput Mech, (2016) 1-12.

[20] A. Foroozmehr, M. Badrossamay, E. Foroozmehr and S.i. Golabi, Finite element simulation of selective laser melting process considering optical penetration depth of laser in powder bed, Mater. Des., 89 (2016) 255-263.

[21] R. Li, Y. Shi, J. Liu, H. Yao and W. Zhang, Effects of processing parameters on the temperature field of selective laser melting metal powder, Powder Metall. Met. Ceram., 48 (2009) 186-195.

[22] K. Zeng, D. Pal, H. Gong, N. Patil and B. Stucker, Comparison of 3DSIM thermal modelling of selective laser melting using new dynamic meshing method to ANSYS, Mater. Sci. Technol., 31 (2015) 945-956.

[23] A. Hussein, L. Hao, C. Yan and R. Everson, Finite element simulation of the temperature and stress fields in single layers built without-support in selective laser melting, Mater. Des., 52 (2013) 638-647.

[24] V. Šmilauer, E. Catalano, B. Chareyre, S. Dorofeenko, J. Duriez, A. Gladky, J. Kozicki, C. Modenese, L. Scholtès, L. Sibille, J. Stránský and K. and Thoeni, Yade Documentation (Šmilauer V, ed.), The Yade Project, 1st ed., 2010, http://yade-dem.org/doc/.

[25] Flow3D: Version 11.0.1.2: User Manual, FlowScience, Santa Fe, NM, USA, 2014.

[26] K. Guan, Z. Wang, M. Gao, X. Li and X. Zeng, Effects of processing parameters on tensile properties of selective laser melted 304 stainless steel, Mater. Des., 50 (2013) 581-586.

[27] A. Klassen, T. Scharowsky and C. Körner, Evaporation model for beam based additive manufacturing using free surface lattice Boltzmann methods, J. Phys. D: Appl. Phys., 47 (2014) 275303.

[28] M.V. Allmen, Laser-beam interactions with materials: physical principles and applications, 2nd ed., Springer, Berlin, 1987.

[29] M. Rappaz, S.A. David, J.M. Vitek and L.A. Boatner, Development of microstructures in Fe-15Ni-15Cr single crystal electron beam welds, Metall. Trans. A, 20 (1989) 1125-1138.

[30] S. Kou, Welding Metallurgy, 2nd ed., John Wiley \& Sons, Inc., New York, 2003.

[31] M. Gäumann, C. Bezençon, P. Canalis and W. Kurz, Single-crystal laser deposition of superalloys: processing-microstructure maps, Acta Mater., 49 (2001) 1051-1062.

[32] J.D. Hunt, Steady state columnar and equiaxed growth of dendrites and eutectic, Mater. Sci. Eng., 65 (1984) 75-83. 
[33] R.R. Dehoff, M.M. Kirka, W.J. Sames, H. Bilheux, A.S. Tremsin, L.E. Lowe and S.S. Babu, Site specific control of crystallographic grain orientation through electron beam additive manufacturing, Mater. Sci. Technol., 31 (2015) 931-938.

[34] M. Ramsperger, R.F. Singer and C. Körner, Microstructure of the nickel-base superalloy CMSX-4 fabricated by selective electron beam melting, Metall. Mater. Trans. A, 47 (2016) 1469-1480.

[35] N.J. Harrison, I. Todd and K. Mumtaz, Reduction of micro-cracking in nickel superalloys processed by selective laser melting: A fundamental alloy design approach, Acta Mater., 94 (2015) 59-68.

[36] Y. Lee, M. Nordin, S. Babu and D. Farson, Effect of fluid convection on dendrite arm spacing in laser deposition, Metall. Mater. Trans. B, 45 (2014) 1520-1529.

[37] J.J. Blecher, T.A. Palmer and T. DebRoy, Solidification map of a nickel-base alloy, Metall. Mater. Trans. A, 45 (2014) 2142-2151.

[38] Z. Gao and O.A. Ojo, Modeling analysis of hybrid laser-arc welding of single-crystal nickel-base superalloys, Acta Mater., 60 (2012) 3153-3167.

[39] X. Lin, T.M. Yue, H.O. Yang and W.D. Huang, Laser rapid forming of SS316L/Rene88DT graded material, Mater. Sci. Eng., A, 391 (2005) 325-336.

[40] R. Trivedi, Interdendritic spacing: Part II. A comparison of theory and experiment, Metall. Trans. A, 15 (1984) 977-982.

[41] W. Kurz and D.J. Fisher, Dendrite growth at the limit of stability: tip radius and spacing, Acta Metall., 29 (1981) 11-20.

[42] L. Li and R.A. Overfelt, Influence of directional solidification variables on the cellular and primary dendrite arm spacings of PWA1484, J. Mater. Sci., 37 (2002) 3521-3532.

[43] W. Wang, P.D. Lee and M. McLean, A model of solidification microstructures in nickel-based superalloys: predicting primary dendrite spacing selection, Acta Mater., 51 (2003) 2971-2987. 